\title{
Drogas ilegales en escolares de Lima y Callao: factores familiares asociados a su consumo
}

\author{
Illegal drugs in Lima and Callao high schools: family factors associated with the consumption
}

\author{
César Gutiérrez ${ }^{1,2}$, Hans Contreras ${ }^{3}$, Delia Trujillo ${ }^{4}$, Eduardo Haro ${ }^{4}$, José Ramos-Castillo 5 \\ ${ }^{1}$ Departamento Académico de Medicina Preventiva y Salud Pública, Facultad de Medicina, Universidad Nacional Mayor de San Marcos. Lima, Perú. \\ ${ }^{2}$ Sección de Epidemiología, Instituto de Medicina Tropical Daniel A. Carrión, Facultad de Medicina, Universidad Nacional Mayor de San Marcos. Lima, Perú. \\ ${ }^{3}$ Sociedad Estudiantil para el Fomento de la Investigación y las Neurociencias, Facultad de Medicina, Universidad Nacional Mayor de San Marcos. Lima, Perú. \\ ${ }^{4}$ Comisión Nacional para el Desarrollo y Vida sin Drogas (Devida). Lima, Perú. \\ ${ }^{5}$ Médico-Cirujano, Facultad de Medicina, Universidad Nacional Mayor de San Marcos. Lima, Perú.
}

\begin{abstract}
Resumen
Introducción: El consumo de drogas es un problema de salud pública a nivel mundial; se viene incrementando en países en vías de desarrollo. Las drogas ilegales, como marihuana e inhalantes (terokal, PVC, benzina y/o acetona), son consumidas cada vez a edades más tempranas. Objetivo: Determinar las características epidemiológicas, personales, escolares y los factores de tipo familiar (involucramiento de los padres con las actividades de sus hijos) que se asocian con el consumo de marihuana e inhalantes por los estudiantes de secundaria de Lima Metropolitana y Callao. Diseño: Estudio analítico y transversal. Lugar: Lima Metropolitana y Callao. Participantes: Cinco mil seiscientos cuarenta y siete estudiantes de $2^{\circ}, 4^{\circ}$ y $5^{\circ}$ año de secundaria. Intervenciones: Revisión y análisis de la base de datos del estudio nacional "Prevención y consumo de drogas en estudiantes de secundaria", llevado a cabo por la Comisión Nacional para el Desarrollo y Vida sin Drogas, el año 2005. Resultados: La prevalencia de vida de consumo de drogas ilegales en estudiantes de secundaria de Lima Metropolitana y Callao fue de 5,9\% (marihuana) y 4,8\% (inhalantes). El consumo de marihuana fue más frecuente en hombres $(7,6 \%)$ que en mujeres $(2,71 \%), p<0,001$. El consumo de marihuana e inhalantes fue significativamente más frecuente $(p<0,001)$ en quienes no conviven con sus padres, en quienes tienen dos o más antecedentes de fracaso escolar, en quienes tienen frecuentes problemas de comportamiento en el colegio y en quienes se "hacen la vaca' (ausentismo escolar). El tipo de colegio (público o privado) no se asoció significativamente con el antecedente de consumo de marihuana e inhalantes. Los factores de riesgo hallados para el consumo de marihuana e inhalantes fueron: tener algún problema de comportamiento o disciplina durante los estudios, dos o más antecedentes de fracaso escolar y 'hacerse la vaca'. Entre los factores de involucramiento familiar, fueron factores protectores: el conocimiento acerca de la ubicación del hijo por parte de los padres luego de la salida del colegio o en los fines de semana, el control de los padres sobre los programas de televisión que mira el estudiante, el comer junto a los padres entre 4 a 7 días a la semana y el informar a sus padres por parte del adolescente sobre el lugar donde sale en las tardes o fines de semana. Conclusiones: Existe una mayor frecuencia de consumo de drogas entre quienes tienen antecedentes de fracaso e indisciplina escolar así como disfunción familiar. Los valores positivos de participación de la familia en las actividades de sus hijos constituyen factores protectores frente al consumo de estas sustancias; el rol familiar es trascendental en la formación del adolescente.
\end{abstract}

Palabras clave: Trastornos relacionados con sustancias; salud escolar; factores de riesgo; salud pública.

\section{Abstract}

Introduction: Drug abuse is a public health problem worldwide and has increased in developing countries. Illegal drugs such as marihuana and inhalants (terokal, PVC, benzene and/or acetone) are consumed increasingly at younger ages. Objectives: To determine the epidemiology, personal, scholastic and familial factors (parental involvement with their children's activities) associated to marihuana and inhalants use by high school students in Metropolitan Lima and Callao. Design: Analytical and transversal study. Setting: Metropolitan Lima and Callao. Participants: Five thousand six hundred and forty-seven high school 2nd, 4th and 5th year students. Interventions: Review and analysis of the "Prevention and drug use by high school students" national study database conducted by the National Commission for Development and Life without Drugs 2005. Results: Lifetime prevalence of illicit drug use among secondary school students in Metropolitan Lima and Callao was 5,9\% (marihuana) and 4,8\% (inhalants). Marihuana use was more common in men $(7,6 \%)$ than in women $(2,71 \%)$, $p$ $<0,001$. Marihuana and inhalants were used significantly more frequently ( $p$ $<0,001$ ) by those who did not live with their parents, had had two or more school failures, having behavior problems in school and those "playing hookey" (truancy). The type of school (public or private) was not significantly associated with history of either marihuana or inhalants. Risk factors found for marihuana and inhalants were having some behavioral or discipline problems during their studies, two or more antecedents of school failure and 'playing hookey'. Among family involvement protective factors we found: parental knowledge on child location after classes or on weekends, parental control on television watching, parents eating with the child 4 to 7 days a week, and the teenager informing his parents where he went in evenings or weekends. Conclusions: Frequency of drug abuse increased among children with history of school failure and indiscipline and family dysfunction. Family involvement in children's activities represents a protective factor against drug abuse; the family has an extremely significant role in shaping the adolescent.

Key words: Substance-related disorders; school health; risk factors; public health.

\section{INTRODUCCIÓN}

La adolescencia es un periodo del desarrollo personal en que se forma el componente conativo-volitivo de la conciencia y el carácter de la personalidad; dura aproximadamente hasta los 18 años de edad, dependiendo del grado de desarrollo económico, la clase social y las condiciones de vida de la familia o comunidad ${ }^{(1)}$.

El consumo de drogas es un problema de salud pública a nivel mundial, siendo la droga ilegal de mayor consumo la marihuana. El consumo de dicha sustancia se hace cada vez a edades más tempranas y se ha incrementado tanto en países desarrollados como en vías de desarrollo. Asimismo, el consumo de marihuana se encuentra asociado a una mayor probabilidad de fracaso escolar, menores logros académicos y reducción de los años de educación, dependiendo de la edad de inicio ${ }^{(2)}$. El Perú no escapa a esa realidad, dado que las dos sustancias más usadas 
en población escolar adolescente son la marihuana y los inhalantes (terokal, PVC, benzina y/o acetona).

Las drogas (legales e ilegales), en general, afectan la capacidad volitiva, interfieren con el proceso cognitivo, contribuyen a la presencia de trastornos del humor; incrementan el riesgo de accidentes, muerte o suicidio; mantienen el ciclo de la pobreza y contribuyen al subdesarrollo. El consumo de drogas puede destruir la integridad y cohesión familiar debido al estrés de los padres; genera también una pérdida de horas de trabajo, debido a una disminución en la productividad y por tanto una disminución del ingreso familiar (3). Además, el uso de sustancias durante la adolescencia puede repercutir en altos costos en términos de cuidados de la salud, tratamiento y servicios de salud mental, así como mayores índices de crímenes juveniles ${ }^{(4)}$. La morbilidad adolescente es mayor cuando se asocia al consumo de drogas ${ }^{(5)}$; así, se ha determinado una fuerte asociación entre trastornos mentales y el uso de drogas $(4,6,7)$.

Siguiendo la tendencia mundial, en nuestro país se evidencia un ascenso constante en las tasas de consumo de drogas ilegales. Según el estudio nacional "Prevención y consumo de drogas en estudiantes de secundaria - 2005" llevado a cabo por la Comisión Nacional para el Desarrollo y Vida Sin Drogas (Devida), en Lima y Callao el consumo de marihuana e inhalantes fueron fenómenos prevalentes. Así, 6 de cada 100 adolescentes entrevistados refirieron haber consumido marihuana y, de ellos, 2 afirmaron haberlo hecho en el transcurso de los últimos 30 días; 5 de cada 100 refirieron haber probado inhalantes y, de ellos, 1 afirmó haberlo hecho en el transcurso de los últimos 30 días. La marihuana $(5,5 \%)$ y los inhalantes $(4,9 \%)$ fueron las drogas de mayor consumo en la población adolescente de Lima y Callao; le siguieron, en orden de frecuencia, cocaína $(2,3 \%)$, pasta básica de cocaína $(1,6 \%)$ y éxtasis $(1,2 \%)^{(8)}$.

El consumo de drogas legales (alcohol y tabaco) e ilegales (marihuana, pasta básica de cocaína y éxtasis), en nuestro país, se ha incrementado durante la última década; sobre todo en personas de 12 a 64 años que residen en zonas urbanas ${ }^{(9)}$.
Según CEDRO, en nuestro país, el año 2003, la prevalencia de vida de consumo de alcohol y tabaco en la población general fue $87,9 \%$ y $63,4 \%$, respectivamente; en la población entre 12 y 18 años, las estimaciones para esas mismas drogas fueron $61,6 \%$ y $31,4 \%$. En el caso del consumo de drogas ilegales, la prevalencia fue $8 \%$ para año 2001 y $11,1 \%$ el 2003 ; esta tendencia ascendente fue más importante en la población entre 12 y 18 años, entre quienes esta prevalencia se triplicó en el mismo período: de 1,6\% el 2001 a 4,9\% el $2003^{(10)}$.

Los estudios realizados por el Instituto Especializado de Salud Mental en Lima Metropolitana, en tres ciudades de la sierra y en tres ciudades de la selva, proporcionaron información sobre el consumo de drogas en población adolescente (12 a 17 años $)^{(11-13)}$. En general, en estos estudios se observó que los varones consumieron más drogas que las mujeres, que el alcohol fue la droga legal que más se consumía: 68,4\% en Lima Metropolitana, $66,4 \%$ en la sierra y $68,1 \%$ en la selva; mientras que la prevalencia en el último mes, para estas mismas drogas, fue $21,6 \%$, $14,2 \%$ y $16 \%$, respectivamente. La prevalencia de vida de consumo de drogas ilegales fue 2,6\% en Lima Metropolitana, $0,8 \%$ en la sierra y $1,6 \%$ en la selva; la droga ilegal más consumida en las tres regiones fue la marihuana. También, se observó que la edad de inicio del consumo de drogas en los adolescentes fue entre 13 y 14 años en Lima Metropolitana, entre 11 y 14 años en la sierra y entre 13 y 14 años en la selva.

En el tema de la drogadicción, la población escolar primaria y secundaria constituyen grupos importantes sobre el cual se presta especial atención, tanto por su vulnerabilidad como por las oportunidades de prevención que ofrece. En nuestro país se han realizado algunos estudios en dichas poblaciones, con la finalidad de conocer la magnitud del problema de consumo de drogas. Así, entre julio y diciembre de 1998, se realizó un estudio que entrevistó a 8213 estudiantes de primaria del país; el estudio evidenció que la prevalencia de vida de consumo de alcohol fue $10,6 \%$, tabaco $3,4 \%$, inhalantes $0,11 \%$ y de marihuana 0,07\%; la edad promedio de inicio de consumo de las diferentes drogas fueron: bebidas alcohólicas 8,78 años, tabaco 9,29 años, inhalantes 9,11 años y marihuana 9,67 años ${ }^{(14)}$. También, se ha realizado investigaciones en estudiantes de secundaria; el "Estudio de Línea Base del Programa de Promoción para una Vida sin Drogas (Segunda Fase)", realizado por el Ministerio de Educación, reveló las siguientes prevalencias de vida de consumo de drogas: bebidas alcohólicas 52,2\%, tabaco $34,7 \%$, inhalantes $3,8 \%$ y marihuana $3,6 \%$. Según estos reportes, la mitad de la población escolar secundaria había consumido alguna vez bebidas alcohólicas y, entre la tercera parte y la mitad, tabaco. En el caso de drogas ilegales, la marihuana y los inhalantes fueron las que con mayor frecuencia habían sido consumidas al menos una vez en la vida ${ }^{(15)}$.

Los consumidores de estas drogas (marihuana e inhalantes) constituyen, para la presente investigación, un grupo importante a focalizar y determinar sus características epidemiológicas, así como los factores de involucramiento y participación familiar que se hallan asociados a su consumo, dado el importante rol en la formación de valores que los padres cumplen en el entorno familiar. Es así que el objetivo del presente estudio fue determinar las características epidemiológicas, características personales, antecedentes escolares y los factores de tipo familiar (involucramiento de los padres con las actividades de sus hijos) que se asocian con la prevalencia de vida de consumo de marihuana e inhalantes de los adolescentes estudiantes de secundaria de Lima Metropolitana y el Callao. Nuestros resultados pretenden contribuir al trabajo preventivo promocional con la difusión de medidas específicas a considerar en el entorno familiar, entendiendo a la familia como el agente rector de la conducta y espiritualidad humana ${ }^{(16)}$.

\section{MÉTODOS}

Se realizó un estudio analítico y transversal, utilizándose para ello la base de datos del estudio nacional "Prevención y consumo de drogas en estudiantes de secundaria", llevado a cabo por la Comisión Nacional para el Desarrollo y Vida sin Drogas (Devida, 2005). Este fue el primer estudio realizado en nuestro medio 
que presentó información confiable sobre la situación del consumo de drogas en estudiantes de secundaria a nivel nacional. Dicho estudio se realizó el año 2005, en una muestra estratificada y sistemática de 60285 estudiantes de ambos géneros, de $2^{\circ}, 4^{\circ}$ y $5^{\circ}$ año de secundaria, de colegios públicos y privados, y cuyas edades coincidían con las de inicio, plenitud y término de la adolescencia; el estudio cubrió un total de 50 ciudades del Perú, incluyendo Lima y Callao ${ }^{(8)}$.

Para el presente estudio, utilizando dicha base de datos, se seleccionó una muestra de 5647 estudiantes de $2^{\circ}, 4^{\circ}$ y $5^{\circ}$ año de secundaria que tenían como lugar de residencia Lima y Callao, según la población estipulada en nuestro objetivo.

Las variables estudiadas en la presente investigación fueron las incluidas en el estudio de Devida ${ }^{(8)}$ : edad, sexo, año de estudio $\left(2^{\circ}, 4^{\circ}\right.$ o $\left.5^{\circ}\right)$, prevalencia de vida de consumo de drogas (marihuana $\mathrm{e}$ inhalantes), convivencia con los padres (con ninguno, con al menos un familiar directo, con ambos padres), tipo de colegio (público o privado), plan de vida al terminar el colegio (carrera universitaria, carrera técnica, fuerzas armadas y policiales, no trabajar), antecedente de fracaso escolar (ninguno, uno, dos o más), problemas de comportamiento o disciplina durante los estudios (nunca, poco, frecuentemente). Respecto a la participación e involucramiento de los padres en las actividades de sus hijos, se estudió las siguientes variables: conocimiento de la ubicación por parte de los padres luego de la salida del colegio o en los fines de semana (nunca, a veces, siempre), control de los padres sobre los programas de televisión (sí, no), atención de los padres sobre el desempeño del estudiante en el colegio (nada, poco, mucho), días de la semana que come el estudiante junto a los padres (nunca, uno a tres días, cuatro a siete días), control de la hora de llegada por parte de los padres luego de salidas los fines de semana (sí, no), el informar a sus padres por parte del adolescente sobre el lugar donde sale en las tardes o fines de semana (sí, no), y conocimiento de los padres acerca de los amigos del estudiante (bastante, más o menos, poco).
Se definió prevalencia de vida del consumo de drogas al porcentaje de la muestra que declaró haber usado o probado una sustancia psicoactiva determinada por lo menos una vez en su vida. Con ello no se intentó determinar las motivaciones que llevan al adolescente a consumir estas sustancias, que pueden ir desde la simple curiosidad a la necesidad de satisfacer la apetencia derivada de la dependencia.

Se utilizó estadística descriptiva para la presentación de datos. Las variables cuantitativas fueron analizadas con medidas de tendencia central y de dispersión. Se analizó las variables cualitativas según prevalencias y proporciones. Se realizó un análisis bivariado, utilizando la prueba de Kolmogorov-Smirnov, para la determinación de la distribución normal de las variables. Asimismo, se utilizó las pruebas paramétricas t de student, Anova, y las pruebas no paramétricas Ji-cuadrado, U de Mann-Whitney o Kruskal-Wallis, según correspondiera. Se realizó un análisis multivariado de regresión logística para la determinación de las asociaciones de prevalencia de vida de consumo de drogas y algunas variables determinantes, como la participación de los padres en las actividades de sus hijos, a través de la medición de OR (odds ratio). Todos los cálculos fueron realizados con el programa estadístico SPSS v.15. Se consideró como resultados significativos aquellos con valores de $p$ menores de 0,05.

\section{RESULTADOS}

La muestra total estudiada fue de 5647 estudiantes, cuyo promedio de edad fue $14,95 \pm 1,51$ (rango: $11-22$ ) años, $51,6 \%$ de sexo masculino y $48,4 \%$ de sexo femenino; $74,9 \%$ provenía de colegios públicos. El 50,5\% vivía en Lima y el 49,5\% en el Callao. El 65,3\% refirió vivir con ambos padres, $28 \%$ vivía solamente con uno de ellos, $6,7 \%$ no vivía con ninguno de ellos.

La prevalencia de vida de consumo de marihuana fue $5,9 \%$ (328 casos); la edad promedio de inicio en el consumo de marihuana fue 14,3 $\pm 1,62$ (rango: 10-18) años. La prevalencia de vida de consumo (aspiración) de inhalantes (terokal, PVC, benzina y/o acetona) fue 4,8\% (268 casos); la edad promedio de inicio de dicho evento (aspiración de inhalantes) fue 13,2 $\pm 1,76$ (rango: 10-18) años. Según sexo, el consumo de marihuana fue más frecuente en hombres $(7,60 \%)$ que en mujeres $(2,71 \%), p<0,001 ; \sin$ embargo, el consumo de inhalantes fue más frecuente en mujeres (5\%) que en hombres $(4,5 \%)$, aunque no hubo diferencia estadísticamente significativa.

La población escolar con antecedente de consumo de marihuana $(5,9 \%)$ fue ligeramente mayor en edad (15,73 vs. 14,9 años, $p<0,001$ ) en relación a los que no consumían dicha droga. Entre quienes consumían marihuana, las siguientes variables se constituyeron más frecuentes respecto a quienes no consumían $(p<0,001)$ : el sexo masculino $(7,6 \%$ de hombres vs. $2,71 \%$ de mujeres), el mayor grado escolar $\left(7,72 \%\right.$ de $4^{\circ}$ año y $7,71 \%$ de $5^{\circ}$ año vs. $2,54 \%$ de $2^{\circ}$ año), la no convivencia con los padres $(8,38 \%$ con ninguno vs $6,13 \%$ con al menos un familiar y 4,69\% con ambos padres), tener dos o más antecedentes de fracaso escolar (13,81\% dos o más veces vs. $9,65 \%$ una vez y $4,70 \%$ ninguna vez), los frecuentes problemas de comportamiento en el colegio $(16,11 \%$ frecuentemente vs. $8,59 \%$ pocas veces y $1,97 \%$ nunca), y 'haberse hecho la vaca' o 'tirado la pera' (14,95\% sí lo hizo vs 3,96\% que no lo hizo). El $10,67 \%$ de los que consumían marihuana refirió deseos de trabajar al terminar el colegio, respecto a quienes desearon estudiar alguna carrera técnica 7,14\% o universitaria 3,99 $(p<0,001)$.

La población escolar con antecedente de aspiración de inhalantes (terokal, PVC, benzina y/o acetona), 4,9\%, tuvo ligeramente mayor en edad (15,1 vs. $14,94$ años, $p<0,05)$ en relación a los que no presentaron el antecedente. Entre los que consumían inhalantes, las siguientes variables se constituyeron más frecuentes respecto a quienes no consumían $(p<0,005)$ : la no convivencia con los padres $(6,9 \%$ con ninguno vs $5,15 \%$ con al menos un familiar y $4,2 \%$ con ambos padres), tener dos o más antecedentes de fracaso escolar (8,8\% dos o más veces vs. $5,1 \%$ una vez y $4,5 \%$ ninguna vez), los frecuentes problemas de comportamiento 
en el colegio $(12,2 \%$ frecuentemente vs. $6,3 \%$ pocas veces y $2,4 \%$ nunca), y 'haberse hecho la vaca' o 'tirado la pera' (10,3\% sí lo hizo vs. $3,6 \%$ que no lo hizo). A diferencia del antecedente de consumo de marihuana, el antecedente de haber aspirado inhalantes en algún momento de la vida no se diferenció ni por año de estudio ni por sexo. El 6,5\% de los que consumía inhalantes refirió deseos de estudiar en la policía o fuerzas armadas al terminar el colegio, respecto a quienes desearon estudiar alguna carrera técnica, $4 \%$, o universitaria, 3,9 $(p<0,001)$.

El tipo de colegio (público o privado) no se asoció significativamente con el antecedente de consumo de marihuana ni de inhalantes, aunque se evidenció un ligero mayor porcentaje de consumidores de ambas drogas provenientes de colegios públicos: $5,92 \%$ público vs. $5,67 \%$ privado, para consumidores de marihuana, y $5 \%$ público vs. $4,2 \%$ privado para consumidores de inhalantes.

Los factores de riesgo asociados al consumo de marihuana fueron: cursar el $4^{\circ}$ año de secundaria (OR: 3,036, IC: $2,16-4,26, p<0,001)$, cursar el $5^{\circ}$ año de secundaria (OR: 3,065, IC: 2,15 4,36, $p<0,001$ ); ambos grados poseían riesgo respecto a los que cursaban $2^{\circ}$ año de secundaria; un antecedente de fracaso escolar (OR: 1,75, IC: 1,13-2,31, $p<0,001)$; tener dos o más antecedentes de fracaso escolar (OR: 2,30, IC: 1,39$3,80, p<0,001)$; tener algunos problemas de comportamiento o disciplina durante los estudios (OR: 3,45, IC: 2,49-4,78, $p<0,001)$; tener frecuentes problemas de comportamiento o disciplina durante los estudios (OR: 4,8, IC: 3,03-4,61, $p<0,001)$; y 'haberse hecho la vaca' o 'tirado la pera' (OR: 2,27, IC: 1,76-2,94, $p<0,001$ ); esto último se halló muy ligado al ausentismo escolar.

Los factores de riesgo asociados al consumo (aspiración) de inhalantes (terokal, PVC, benzina y/o acetona) fueron: tener algunos problemas de comportamiento o disciplina durante los estudios (OR: 2,35, IC: $1,71-3,23, p<0,001)$; tener frecuentes problemas de comportamiento o disciplina durante los estudios (OR: 3,67, IC: 2,29-5,91, $p<0,001)$; y 'haberse hecho la vaca' o 'tirado la pera' (OR: 2,03, IC:
1,52-2,69, $p<0,001)$. Es de resaltar que el sexo masculino constituyó, para esta droga (inhalantes), un factor protector (OR: 0,724, IC: 0,55-0,95, p<0,001).

En la tabla 1 se muestra la prevalencia de vida de consumo de marihuana respecto a las variables de involucramiento familiar de los padres (participación en las actividades de sus hijos). Tal como muestra la tabla, existió una relación inversa entre la frecuencia del consumo de marihuana y las medidas de involucramiento familiar (valores positivos) por parte de los padres; así, cuanto mayor era el indicador de involucramiento, menor era la prevalencia del consumo de marihuana $(p<0,001)$. En cuanto a las medidas de asociación, constituyeron factores protectores frente al consumo de marihuana el conocimiento constante acerca de la ubicación del hijo por parte de los padres luego de la salida del colegio o en los fines de semana, el control de los padres sobre los programas de televisión que miraba el estudiante, el comer junto a los padres entre 4 a 7 días a la semana y el informar a sus padres -por parte del adolescente- sobre el lugar donde salía en las tardes o fines de semana (figura 1).

Tabla 1. Prevalencia de vida de consumo de marihuana entre escolares de secundaria de Lima y Callao, según indicadores de involucramiento y participación familiar.

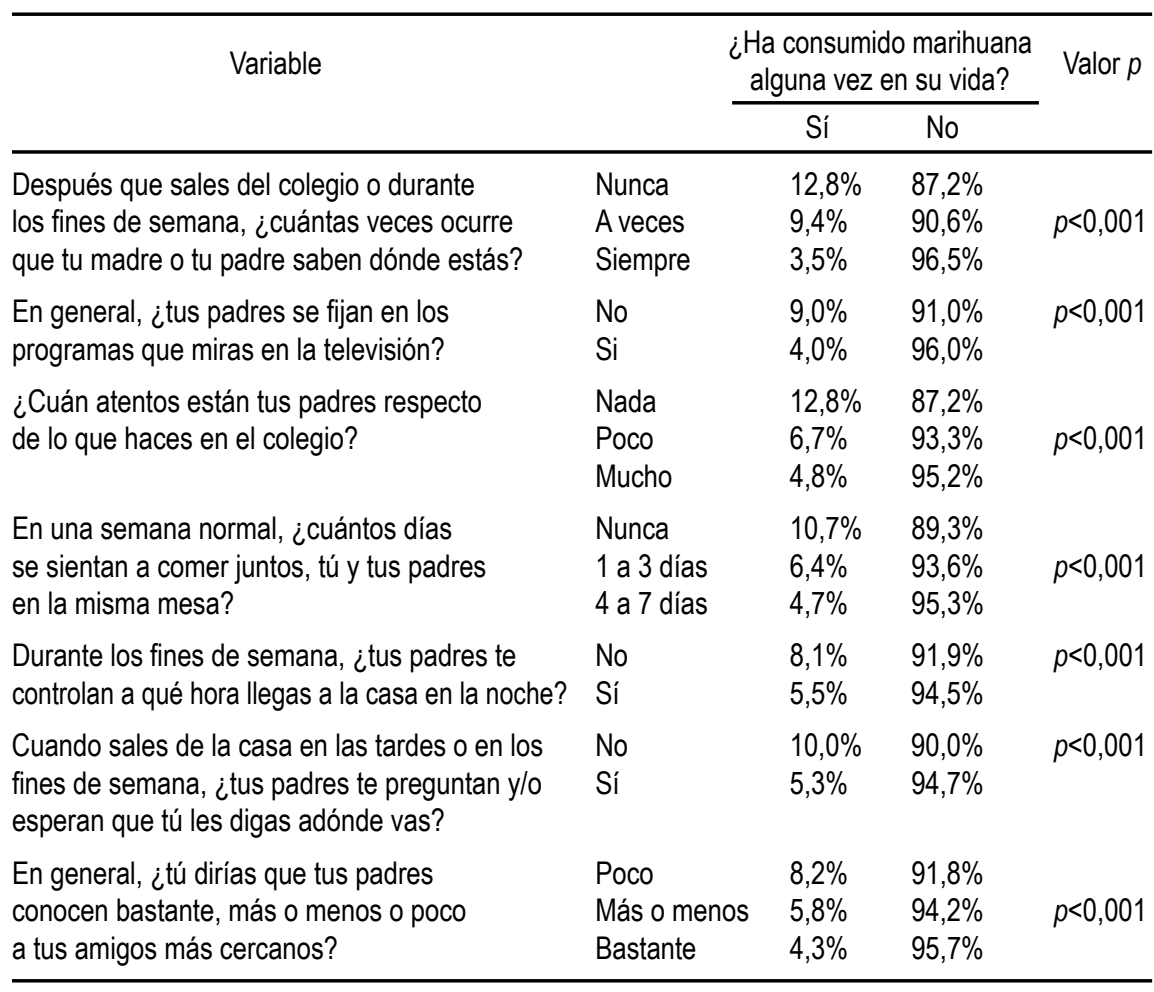

De igual manera, existió una relación inversa en cuanto al consumo de inhalantes y las medidas de involucramiento familiar (valores positivos); así, cuanto mayor fue el indicador de involucramiento, menor era la prevalencia del consumo de inhalantes $(p<0,001)$ (tabla 2). Constituyeron factores protectores frente al consumo de inhalantes: el conocimiento constante acerca de la ubicación del hijo por parte de los padres, luego de la salida del colegio o en los fines de semana, y el comer junto a los padres entre 4 a 7 días a la semana (figura 2).

\section{DISCUSIÓN}

Nuestros hallazgos de prevalencia de vida de consumo de marihuana e inhalantes en la población estudiada concuerdan con los de otros trabajos ${ }^{(11-13,15)}$ y se reafirma en la tendencia hacia el mayor consumo de estas drogas ilegales, con especial énfasis en la población escolar adolescente.

En el grupo con antecedente de consumo de marihuana existió una mayor prevalencia significativa del sexo masculino y de años superiores $\left(4^{\circ}\right.$ y $\left.5^{\circ}\right)$; en el grupo con antecedente de consumo de 
Figura 1. Indicadores de involucramiento y participación familiar, protectores del consumo de marihuana en escolares de secundaria de Lima y Callao.

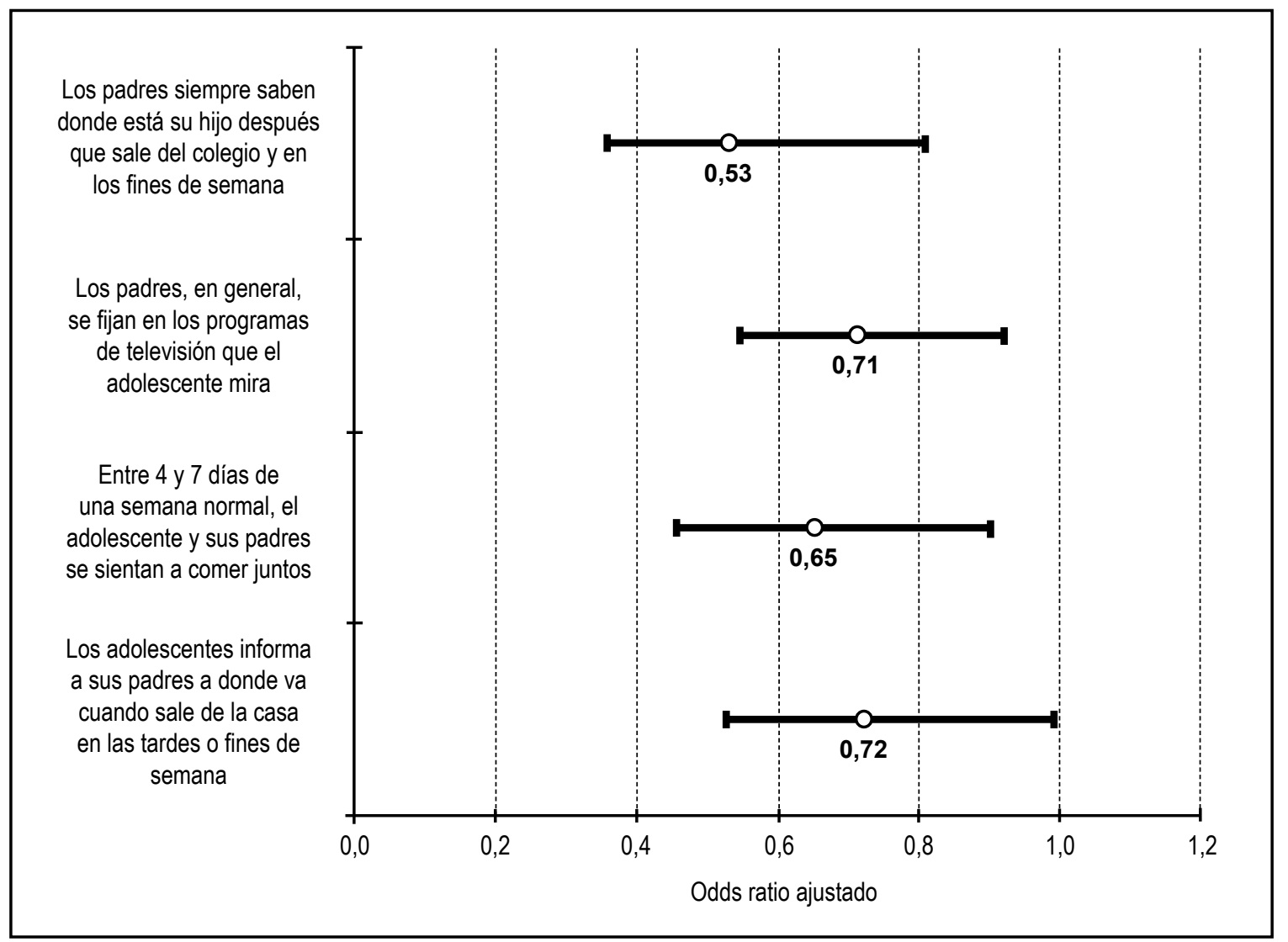

inhalantes, a pesar de ser más prevalente en mujeres y en años inferiores $\left(2^{\circ} \mathrm{y}\right.$ $\left.4^{\circ}\right)$, no hubo una diferencia significativa asociada ni al sexo ni al año de estudio, referente a un mayor consumo de drogas. Este segundo grupo, en relación al grupo de consumo de marihuana, marca una distancia epidemiológica capital: mayor predominio femenino y mayor prevalencia en los alumnos de grados menores. Esta distinción es un rasgo epidemiológico importante, pues, a nuestra consideración, indicaría que los casos de consumo de inhalantes podrían pasar desapercibidos ante los padres de familia y/o los profesores. El incremento del consumo de drogas en este grupo (mujeres de grados secundarios menores) es un fenómeno que se viene dando en la actualidad, según algunos informes. Por otro lado, es muy probable que exista un uso paralelo de inhalantes y marihuana; aquellos con antecedente de haber probado inhalantes presentaron 15,3 (IC: 12,28-19,57, $p<0,001)$ veces más riesgo de haber consumido marihuana. Sakai y colaboradores concluyen, estudiando específicamente el caso de los inhalantes, que se debe prestar especial interés en estos casos, pues se podría tratar de patología múltiple, ya sea de uso de sustancias simultáneas o resultar en patologías, como depresión mayor e intento de suicidio ${ }^{(6)}$. Si a esto le añadimos, como demuestran nuestros resultados, una posiblemente difícil identificación de los casos, la situación de este grupo resulta particularmente riesgosa.

Si bien el tipo de colegio (público o privado) no se asoció significativamente con el antecedente de consumo de marihuana e inhalantes, existió un mayor porcentaje de consumidores provenientes de colegios públicos. Cabe resaltar que son distintas las realidades según sea el antecedente: el consumo de marihuana se relacionó más a una problemática en relación a las normas (mayor prevalencia de problemas de disciplina y comportamiento) y al fracaso escolar, respecto al consumo de inhalantes, donde son mucho menores las frecuencias de estas características.
Dentro de los factores de riesgo hallados para el consumo de marihuana se encontró: cursar el $4^{\circ}$ o $5^{\circ}$ año de secundaria, tener alguno o frecuentes antecedentes de fracaso escolar, tener algunos o frecuentes problemas de comportamiento o disciplina durante los estudios y 'hacerse la vaca' (ausentismo escolar). Para el consumo de inhalantes, los factores de riesgo encontrados fueron: tener algunos problemas de comportamiento o disciplina durante los estudios y 'hacerse la vaca'. Nuestros hallazgos son similares a lo ya descrito en algunos estudios ${ }^{(2,3)}$; sin embargo, es importante resaltar que, si bien pudieran ser desencadenantes para el consumo de drogas, también podrían ser el efecto de la inmersión en el submundo de dichas sustancias, dado que estas llevan a cambios de conducta y personalidad ${ }^{(1)}$.

Más allá de las distinciones específicas, comparten fenómenos comunes, y estos giran principalmente en torno a la disfunción familiar, que se corrobora en 
Tabla 2. Prevalencia de vida de consumo de inhalantes (terokal, PVC, benzina y/o acetona) entre escolares de secundaria de Lima y Callao según indicadores de involucramiento y participación familiar.

\begin{tabular}{|c|c|c|c|c|}
\hline \multirow[t]{2}{*}{ Variable } & & \multicolumn{2}{|c|}{$\begin{array}{l}\text { ¿Ha consumido inhalantes } \\
\text { alguna vez en su vida? } \\
\end{array}$} & \multirow[t]{2}{*}{ Valor $p$} \\
\hline & & Sí & No & \\
\hline $\begin{array}{l}\text { Después que sales del colegio o durante } \\
\text { los fines de semana, ¿cuántas veces ocurre } \\
\text { que tu madre o tu padre saben dónde estás? }\end{array}$ & $\begin{array}{l}\text { Nunca } \\
\text { A veces } \\
\text { Siempre }\end{array}$ & $\begin{array}{l}10,4 \% \\
6,9 \% \\
3,2 \%\end{array}$ & $\begin{array}{l}89,6 \% \\
93,1 \% \\
96,8 \%\end{array}$ & $p<0,001$ \\
\hline $\begin{array}{l}\text { En general, ¿tus padres se fijan en los } \\
\text { programas que miras en la televisión? }\end{array}$ & $\begin{array}{l}\text { No } \\
\text { Sí }\end{array}$ & $\begin{array}{l}6,1 \% \\
4,0 \%\end{array}$ & $\begin{array}{l}93,9 \% \\
96,0 \%\end{array}$ & $p<0,001$ \\
\hline $\begin{array}{l}\text { ¿Cuán atentos están tus padres respecto } \\
\text { de lo que haces en el colegio? }\end{array}$ & $\begin{array}{l}\text { Nada } \\
\text { Poco } \\
\text { Mucho }\end{array}$ & $\begin{array}{l}11,9 \% \\
5,6 \% \\
3,7 \%\end{array}$ & $\begin{array}{l}88,1 \% \\
94,4 \% \\
96,3 \%\end{array}$ & $p<0,001$ \\
\hline $\begin{array}{l}\text { En una semana normal, ¿cuántos días } \\
\text { se sientan a comer juntos, tú y tus padres } \\
\text { en la misma mesa? }\end{array}$ & $\begin{array}{l}\text { Nunca } \\
1 \text { a } 3 \text { días } \\
4 \text { a } 7 \text { días }\end{array}$ & $\begin{array}{l}8,5 \% \\
5,2 \% \\
3,8 \%\end{array}$ & $\begin{array}{l}91,5 \% \\
94,8 \% \\
96,2 \%\end{array}$ & $p<0,001$ \\
\hline $\begin{array}{l}\text { Durante los fines de semana, ¿tus padres te } \\
\text { controlan a qué hora llegas a la casa en la noche? }\end{array}$ & $\begin{array}{l}\text { No } \\
\text { Sí }\end{array}$ & $\begin{array}{l}7,5 \% \\
4,3 \%\end{array}$ & $\begin{array}{l}92,5 \% \\
95,7 \%\end{array}$ & $p<0,001$ \\
\hline $\begin{array}{l}\text { Cuando sales de la casa en las tardes o en los } \\
\text { fines de semana, ¿tus padres te preguntan y/o } \\
\text { esperan que tú les digas adónde vas? }\end{array}$ & $\begin{array}{l}\text { No } \\
\text { Sí }\end{array}$ & $\begin{array}{l}7,2 \% \\
4,4 \%\end{array}$ & $\begin{array}{l}92,8 \% \\
95,6 \%\end{array}$ & $p<0,001$ \\
\hline $\begin{array}{l}\text { En general, ¿tú dirías que tus padres conocen } \\
\text { bastante, más o menos o poco a tus amigos } \\
\text { más cercanos? }\end{array}$ & $\begin{array}{l}\text { Poco } \\
\text { Más o menos } \\
\text { Bastante }\end{array}$ & $\begin{array}{l}3,0 \% \\
4,7 \% \\
7,6 \%\end{array}$ & $\begin{array}{l}97,0 \% \\
95,3 \% \\
92,4 \%\end{array}$ & $p<0,001$ \\
\hline
\end{tabular}

nuestros resultados, dado que los valores positivos de participación de la familia en las actividades de su hijo constituyen factores protectores frente al consumo de marihuana e inhalantes, a resaltar, el conocimiento constante acerca de la ubicación del hijo por parte de los padres luego de la salida del colegio o en los fines de semana, el control de los padres sobre los programas de televisión que mira el estudiante, el comer junto a los padres entre 4 a 7 días a la semana y el informar a sus padres por parte del adolescente sobre el lugar donde sale en las tardes o fines de semana. Por tanto, queda demostrado a partir de nuestros hallazgos, que la participación de los padres en las actividades de sus hijos constituyen factores asociados a una menor probabilidad de consumo de drogas y sobre el cual habría que incidir en las tareas preventivo promocionales, a cargo de las instituciones encargadas de esta problemática, para así poder disminuir la prevalencia de consumo de drogas en escolares. Lo anterior concuerda con lo también encontrado en otros estudios relacionados al tema de drogadicción y que fueron realizados en nuestro medio
$(17,18)$. Galindo y colaboradores, en un estudio que evaluó la vulnerabilidad familiar entre pacientes adictos y no adictos, encontró que las familias de los pacientes adictos tenían como perfil: cohesión familiar baja, dificultad para tomar decisiones y solucionar sus problemas con la participación democrática de todos sus miembros, dificultad en la comunicación entre padres e hijos (especialmente con el padre), escasas rutinas familiares que contribuyeran a un buen nivel de organización familiar, pocas o inexistentes fuentes de apoyo en la familia y en el entorno para el adolescente en problemas, escasa atención en el rendimiento académico de los hijos, un grado alto de insatisfacción de la familia como sistema, entre otros ${ }^{(18)}$. Nuestros resultados confirman dicho planteamiento: la familia tiene un rol importantísimo en el desarrollo de la tes; la estructuración de la personalidad depende en gran medida de la presencia o ausencia de los padres, así como de las características y formas de relación con ellos, sus normas y valores culturales ${ }^{(19)}$. Para efectos de prevención terciaria, la personalidad y conducta de los adolescen- rehabilitación también depende en gran medida del apoyo familiar ${ }^{(20)}$.

La teoría psicobiológica social dialéctica resalta que, más allá del hecho de que exista o no adicción, el antecedente de consumo de drogas pone en manifiesto un problema en la formación de la personalidad ${ }^{(20,21)}$. Esto habrá de reflejarse posteriormente en una ausencia de plan de vida (como demuestran nuestros resultados: el hecho de no saber qué hacer una vez culminados sus estudios secundarios en quienes hubo antecedente de consumo de drogas) y en una larga serie de trastornos (ausentismo escolar 'hacerse la vaca', problemas de conducta, fracaso escolar, entre otros, también demostrados en el presente estudio). Debemos recordar que el plan de vida se estructura, se aprehende, se enseña ${ }^{(1)}$.

A raíz del conocimiento actual, no cabe duda que los factores genéticos son un determinante importante en las adicciones; estudios en gemelos han dado soporte a dicho planteamiento ${ }^{(22)}$. En el presente estudio, más allá de centrarnos en cuestiones epigenéticas, afrontamos el problema del consumo de drogas desde una perspectiva social, resaltando el rol capital que juega la familia como ente organizador de lo que se ha dado en llamar la espiritualidad humana, y que también podemos definir como el aspecto conativo-volitivo de la personalidad. Y aunque en la actualidad no existe algún estudioso de la conducta humana que niegue el papel del entorno, poco se comprende realmente sobre el rol de dicho 'entorno'; esto se debe, pensamos, a la ausencia de una teoría del hombre, de una neurociencia social, que se encargue de estudiar el cerebro del ser humano y no el cerebro del mamífero superior (objetivo por demás central de la neurociencia natural, que se desarrolla con mucho auge actualmente). Por ejemplo, se dice que el ser humano es un ser biopsicosocial y con ello se pretende integrar en un solo ente organizado a lo biológico, lo mental y lo social como capas que se van sumando unas a otras, sin haberse definido el sentido y razón de cada una. La neurociencia resalta una naturaleza bipartita del cerebro: el placer (representado por el sistema límbico) y el control (representado por la corteza frontal). Así, las adicciones son 
Figura 2. Indicadores de involucramiento y participación familiar, protectores del consumo de inhalantes en escolares de secundaria de Lima y Callao.

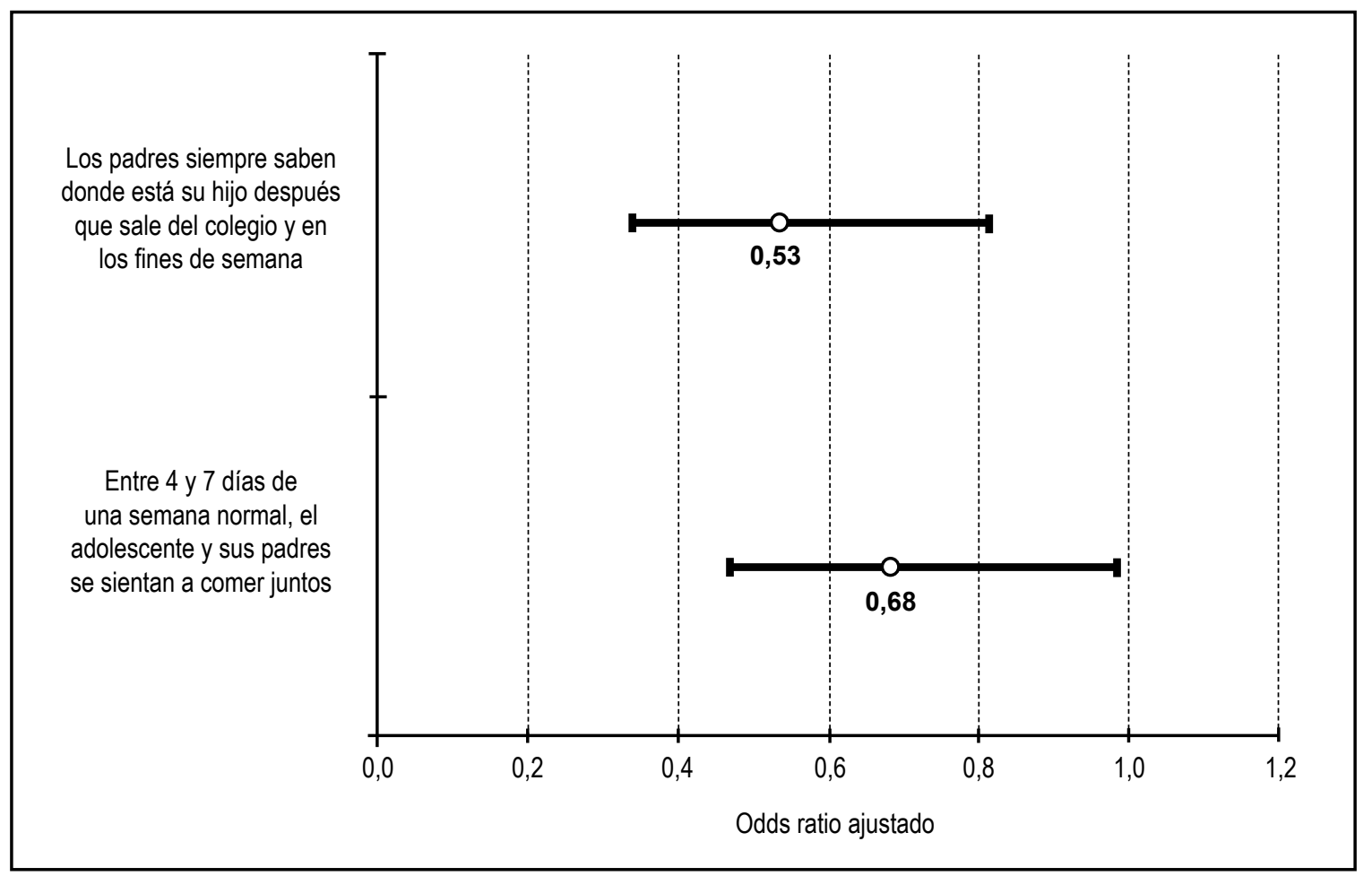

descritas como una imposibilidad en el control de impulsos, se habla de 'neuronas adictas', de una incapacidad en la represión del deseo del placer. Sin embargo, si partimos por una nueva concepción del ser humano, nos referiríamos a los hombres como psiquismos sociales ${ }^{(1)}$; así, el hombre es el reflejo de su sociedad, y por lo tanto, la dialéctica social sería también la dialéctica humana. Con ello, el problema de las adicciones dejaría de centrarse en el cerebro humano y se constituiría en lo que es: un problema social, un problema que para su solución exige no solo cuestiones orgánicas, sino ante todo un nuevo lineamiento de moralidad, una restructuración ética de la sociedad, que bien podría comenzar por la familia, tal como se plantea en el modelo familiar de las adicciones ${ }^{(16)}$.

Evaluar el rol que tienen los agentes sociales, y en especial la familia, sería una forma más amplia de concebir el mundo y sus problemas sociales. La espiritualidad humana (el componente conativovolitivo de la personalidad) y el plan de vida se estructuran, se aprehenden desde la concepción, pero sobre todo durante la adolescencia ${ }^{(1)}$. En ese sentido, vale la pena resaltar un fenómeno poco atendido, pero que viene tomando auge en la investigación contemporánea: la religiosidad; la religión (concebida como una capacidad intrínsecamente humana y de origen eminentemente social) constituye una manera de instauración de los patrones ético-morales entre las personas. Un estudio llevado a cabo en los Estados Unidos se propuso evaluar la religiosidad como factor protector frente al uso de sustancias en jóvenes adultos, encontrando que la religiosidad cumple un efecto protector importante frente al uso de alcohol, consumo de tabaco y consumo de marihuana. Se encontró, por ejemplo, que por cada unidad de incremento en el índice de religiosidad se reducía el uso de alcohol en 9\%, el consumo de tabaco en $13 \%$ y el consumo de marihuana en $20 \%{ }^{(23)}$.

El presente estudio fue diseñado para examinar la influencia de las características demográficas, escolares y familiares del adolescente en el uso de drogas ilegales (marihuana e inhalantes). No han sido consideradas las drogas socialmente aceptadas, que sin lugar a duda participan de modo intenso en la iniciación y con- tinuación de conductas de riesgo, como aquellas referidas al consumo de drogas ilegales. Asimismo, según los últimos reportes de CEDRO (2007) y Devida (2006) sobre consumo de drogas en la población urbana en general, se comprueba un incremento de prevalencia de vida de consumo de marihuana, cocaína y éxtasis comparados con años anteriores ${ }^{(9,24)}$ Cabe mencionar, que si bien la presente investigación se realizó en base al estudio de prevención y consumo de drogas en estudiantes de secundaria (Devida 2005), según el II estudio nacional sobre prevención y consumo de drogas en estudiantes de secundaria del año 2007, también realizado por Devida (25), se halló un incremento del consumo de drogas, sobre todo aquellas que tienen como componente a la cocaína.

Concluimos, según nuestros hallazgos, que la prevalencia de vida del consumo de drogas ilegales en estudiantes de secundaria de Lima Metropolitana y el Callao fue de $5,9 \%$ de marihuana y $4,8 \%$ de consumo de inhalantes. Asimismo, el consumo de marihuana fue más frecuente en varones, en aquellos de grados académicos superiores ( $4^{\circ}$ y $5^{\circ}$ año de secundaria), en 
quienes no conviven con sus padres, en quienes tienen dos o más antecedentes de fracaso escolar, en quienes tienen frecuentes problemas de comportamiento en el colegio, y en quienes se 'hacen la vaca' (ausentismo escolar). El tipo de colegio (público o privado) no se asoció significativamente con el antecedente de consumo de marihuana e inhalantes. El consumo de inhalantes fue más frecuente en quienes no conviven con sus padres, en quienes tienen dos o más antecedentes de fracaso escolar, en quienes tienen frecuentes problemas de comportamiento en el colegio, y en quienes se 'hacen la vaca'. Los factores de riesgo para el consumo de marihuana fueron: cursar el $4^{\circ}$ o $5^{\circ}$ año de secundaria, tener algunos o frecuentes antecedentes de fracaso escolar, tener algunos o frecuentes problemas de comportamiento o disciplina durante los estudios y 'hacerse la vaca' (ausentismo escolar); para el consumo de inhalantes fueron: tener algunos problemas de comportamiento o disciplina durante los estudios y 'hacerse la vaca'. Entre los factores de involucramiento familiar (participación de los padres en las actividades de sus hijos), fueron factores protectores: el conocimiento constante acerca de la ubicación del hijo por parte de los padres luego de la salida del colegio o en los fines de semana, el control de los padres sobre los programas de televisión que mira el estudiante, el comer junto a los padres entre 4 a 7 días a la semana y el informar a sus padres por parte del adolescente sobre el lugar donde sale en las tardes o fines de semana. Nuestros resultados revelan, así, el trascendental rol que tienen los padres en la formación del adolescente; un rol que, sin lugar a dudas, no se reduce a la adolescencia, pero que debe centrarse en esta etapa en guiar la estructuración del carácter.

\section{REFERENCIAS BIBLIOGRÁFICAS}

1. Ortiz Cabanillas, P. Desarrollo formativo de la actividad psíquica personal. Paediatrica. 2004;6(1):29-41.

2. Van Ours J, Williams J. Cannabis use and mental health problems [Internet]. Rochester, New York: Social Science Research Network; [citado el
20-09-2009]. Disponible en: http://ssrn.com/ abstract $=1436901$

3. Casey B, Rebecca J, Todd H. The adolescent brain. Ann N Y Acad Sci. 2008;1124:111-26.

4. Goodman E, Huang B. Socioeconomic status, depressive symptoms, and adolescent substance use. Arch Pediatr Adolesc Med. 2002;156(5):44853.

5. Paniagua H, García S, Castellano G, Serrallé R, Redondo C. Consumo de tabaco, alcohol y drogas no legales entre adolescentes y relación con los hábitos de vida y el entorno. Anales Españoles de Pediatría. 2001;55(2):121-8.

6. Sakai JT, Hall SK, Mikulich-Gilbertson SK, Crowley TJ. Inhalant use, abuse, and dependence among adolescent patients: commonly comorbid problems. J Am Acad Child Adolesc Psychiatry. 2004;43(9):1080-8.

7. Graves K, Fernandez M, Shelton T, Frabutt J, Williford A. Risk and protective factors associated with alcohol, cigarette, and marihuana use during adolescence. J Youth Adolesc. 2005;34(4):37987.

8. Comisión Nacional para el Desarrollo y Vida sin Drogas (DEVIDA). Estudio Nacional: Prevención y Consumo de Drogas en Estudiantes de Secundaria 2005 [Internet]. Lima: Devida; 2005 [citado el 25-09-2009]. Disponible en http://www.Devida. gob.pel

9. Centro de Información y Educación para la Prevención del Abuso de Drogas (CEDRO). Epidemiología de drogas en población urbana [Internet]. Lima: Cedro; 2007 [citado el 12-102009]. Disponible en: http://www.cedro.org.pe/ publicaciones/pesmonog.htm

10. Centro de Información y Educación para la Prevención del Abuso de Drogas CEDRO. Epidemiología de drogas en la población urbana [Internet]. Lima: Cedro; 2004 [citado el 12-102009]. Disponible en: http://www.cedro.org.pe/ publicaciones/pesmonog.htm

11. Instituto Especializado de Salud Mental. Estudio Epidemiológico Metropolitano en Salud Mental 2002. Anales de Salud Mental. 2002;18(1-2).

12. Instituto Especializado de Salud Mental. Estudio Epidemiológico en Salud Mental en la Sierra Peruana 2003. Anales de Salud Mental. 2003;19(1-2).

13. Instituto Especializado de Salud Mental. Estudio Epidemiológico en Salud Mental en la Selva Peruana 2004. Anales de Salud Mental. 2005;21(1-2).

14.Sánchez L, Ramos J. Perfeccionamiento de un modelo explicativo multivariable relativo a la prevalencia de vida de uso de sustancias psicoactivas en la población de estudiantes de primaria de menores en el Perú. Psicoactiva. 2001;19:47-61.

15. Ministerio de Educación del Perú. Estudio de Línea Base del Programa de Promoción para una
Vida sin Drogas (Segunda Fase). Lima: Minedu; 2005.

16. Nizama-Valladolid, M. Guía para el manejo familiar de las adicciones: Modelo Familiar (afronte holístico de las adicciones). Lima: Fondo Editorial Universidad Alas Peruanas; 2001.

17. Silva I. Factores protectores y de riesgo asociados al uso de alcohol en adolescentes hijos de padres alcohólicos, en el Perú. Rev Latino-Am Enfermagem. 2004;(12):359-68.

18. Galindo J, Osso L, Mormontol W, Rodriguez L. Vulnerabilidad familiar al consumo de drogas en familiares adolescentes. Revista de Psiquiatría y Salud Mental Hermilio Valdizán. 2005;5(1):325.

19. Sánchez R, Guisa V, Cedillo A, Pascual Y. Imagen del padre en varones usuarios de sustancias. Actas Esp Psiquiatr. 2002;30(1):36-42.

20.Dakof, Gayle A, Tejeda M, Liddle H. Predictors of engagement in adolescent drug abuse treatment. J Am Acad Child Adolesc Psychiatry. 2001;40(3):274-81.

21. Ortiz Cabanillas, P. Educación y formación de la personalidad. Lima: Fondo Editorial-UCH; 2008.

22. Rhee SH, Hewitt JK, Young SE, Corley RP, Crowley TJ, Stallings MC. Genetic and environmental influences on substance initiation, use, and problem use in adolescents. Arch Gen Psychiatry. 2003;60(12):1256-64.

23. Rostosky S, Danner F, Riggler E. Is religiosity a protective factor against substance use in young adulthood? Only if you're straight! J Adolesc Health. 2007;40(5):440-7.

24. Comisión Nacional para el Desarrollo y Vida sin Drogas (DEVIDA). III Encuesta Nacional de Consumo de Drogas en Población General. Lima: Devida; 2006 [citado el 12-10-2009]. Disponible en http://www.Devida.gob.pe/

25. Comisión Nacional para el Desarrollo y Vida sin Drogas (DEVIDA). Il Estudio Nacional: Prevención y consumo de drogas en estudiantes de secundaria 2007 [Internet]. Lima: Devida; 2007 [citado el 12-10-2009]. Disponible en http://www.Devida. gob.pe/

Manuscrito recibido el 28 de octubre de 2009 y aceptado para publicación el 7 de diciembre de 2009.

\section{Correspondencia:}

Dr. César Gutiérrez Villafuerte

Sección de Epidemiología, Instituto de Medicina

Tropical Daniel A. Carrión - UNMSM

José Santos Chocano 199

Callao 02, Perú.

Correo-e: cgutierrezv@epiredperu.net 